\title{
A SALTATIONIST APPROACH FOR THE EVOLUTION OF HUMAN COGNITION AND LANGUAGE
}

\author{
SUSAN J. LANYON \\ Cognitive Science Program, School of History and Philosophy of Science, \\ University of New South Wales, Sydney, NSW 2052, Australia \\ susanlanyon@bigpond.com
}

\begin{abstract}
The debate over the evolution of an innate language capacity seems to divide into two principle camps. The neo-Darwinian approach generally argues that human psychological modules, including the language faculty, must have arisen gradually and incrementally having been honed by natural selection. Thus Pinker, when theorizing about language evolution, sees "no reason to doubt that the principle explanation is the same as for any other complex instinct or organ, Darwin's theory of natural selection" (Pinker, 1994, 333). However, as Knight et al. (2000) have pointed out, little attention has been paid by the neo-Darwinian approach to address the causes of the emergence of novelty. The saltationist approach gleans much of its evidence from the archaeological and paleontological record, which is interpreted as unsupportive of the neo-Darwinian paradigm. Jackendoff (1999) accuses those who do not accept that language arose gradually through natural selection as having been "forced to devalue evolutionary argumentation". Jackendoff's concern seems to stem from the view that there is only one way that evolution can proceed, through gradual change driven by natural selection. My concern is for the neglect of the vast amount of evidence supporting the theory that modern humans did not emerged in a gradual, step-wise fashion, so there is no reason to believe that cognition and language evolved in this manner. Here I argue that hominins evolved through major evolutionary leaps, which may have numbered only two or three significant mutation "events". Neoteny (the retention of infant or juvenile growth rates) appears to have been a major force in the evolution of our primate ancestors and this process can explain the sudden emergence of many of the traits that define what it means to be human. Further evidence from the fossil and archaeological record supports a "sudden" emergence of human cognition and language.
\end{abstract}

\section{Evolution - Gradual vs Saltational}

Evolution by natural selection in the Darwinian tradition is the most commonly attested theory for the evolution of modern humans. This theory generally assumes that human evolution, including the evolution of the brain, has progressed incrementally as a trend toward complexity. Regardless of the "unit of selection", be it the phenotype or an individual gene, the neo-Darwinian model emphasizes gradualism. Numerous neo-Darwinian evolutionary accounts have proposed that traits like bipedalism, a large brain, reduced dentition, language, tool use and other characteristics of hominins, must have evolved together while maintaining some sort of feedback mechanism that has "fine tuned" or "gradually" adapted each 
characteristic feature to a changing environment.

Thus Pinker has argued that since the emergence of bipedalism a few million years ago, and with the hands freed, subsequent species ratchet upward, click by click, in the features that distinguish us, like the size of the brain and the sophistication of tools. Pinker has interpreted the fossil record under a gradualist scenario with a reduction in the brow ridges, the teeth and the jaw because "tools and technology have taken over from teeth" (Pinker, 1997, 200). However, the reduction in size of the ape-like "dagger-like" canines in the first hominins, can be explained by the process of neoteny. The canines are the last teeth to fully form in juvenile chimpanzees and appear to have not reached their full potential in australopithecines, who appear to have partly skipped the juvenile growth spurt that is part of the life-history of other apes. Although there appear to be some subtle differences in developmental pathways between australopithecines and chimpanzees (Kuykendall 2003), it is generally accepted that the life history of this genus was generally ape-like. Moreover, the first stone tools appear around 4 million years after bipedalism and small canines emerged, hardly a "click by click" scenario. The brain of australopithecine did not increase in size from that of a chimpanzee over this vast period of time. It should therefore not be surprising that we cannot find any form of human-like behaviour during this era of hominin evolution.

Following the "gradualist" school, Jackendoff (1999) claims to be able to decompose modern language into partially ordered steps that have evolved incrementally to be finally integrated into the larger combinatorial system. These modules are assumed to be innate, localized and part of our genetic inheritance. His claim that the language faculty exhibits a degree of modularity is not disputed. Jenkins $(2000,145)$, who also questions the gradualist approach, nevertheless accepts that the language faculty seems to contain modules such as a lexicon, a computational component, semantics, morphology, the phonological component, and phonetics. Jackendoff's intuition that any increase in explicit expressive power of the communication system is adaptive, whether for cooperation in hunting, gathering, defense, or for social communication such as gossip, is also not disputed. But, as Bickerton points out, "there is simply not one scintilla of evidence: simply a blind faith that, if evolution is gradual, and we are where we are, we must have got here, far as it may seem, in a series of incremental steps" (Bickerton, 2002, 104). Rather, the saltationist approach to the evolution of the human ancestral line matches closely with the paleontological evidence, whereas the neo-Darwinian approach, is quite contradictory to that evidence.

\section{Evolution through Developmental Change}

Rather than evolving in gradual, incremental steps from an ancestral primate, the hominin clade appears to have arisen due to the heterochronic variation in form during the early stages of development producing the novelty that we see in the first hominins as well as modern humans. Lovejoy et al. (1999) have argued that 
whilst systematic assembly mechanisms for the formation of various functional anatomical parts of the mammalian bauplan appear to be highly conserved, subtle changes in developmental rate or timing can produce profound morphological changes in phenotype. They have shown how a simple dimensional change in the development of the chimpanzee pelvis could have produced the anatomical differences found in australopithecines. Also, the neck of the hominid femur together with the human knee can be explained by a simple heterochronic change in conjunction with cartilage plasticity and habitual bipedal gait.

The affinity of the juvenile form of both chimpanzees and ancestral hominins, as well as the likeness of some $H$. erectus juvenile hominins and modern humans, shows that most of the skeletal traits of the hominin line arose primarily due to changes in developmental timing in the early stages of ontogeny. Neoteny has been a major factor in the evolution of ancestral hominins. It is interesting to note that the first discoveries and classification of Australopithecus africanus by Dart in the first half of the twentieth century, were rejected by many, claiming that these remains were those of a juvenile gorilla.

Chimpanzees are born with the skull positioned on the top of the spinal column, just like human infants. The newborn chimpanzee is bipedal albeit with a bent-knee, bent-hip gait similar to that posited for australopithecine and even perhaps Neanderthals. The skull of the chimpanzee is also similar to that of a human with a flat face, without brow ridges, without cranial crests, same position of orbits, thinness of bone and a high domed forehead. The infant chimpanzee also has a small human-like jaw and palate. All of these human-like features change dramatically during the juvenile growth spurt of the chimpanzee. Penin et al. (2002) have recently confirmed the hypothesis for the neotenic emergence of the human skull shape. Their results show that the adult skull in humans reaches a size-related shape that is equivalent to that of a juvenile chimpanzee but nevertheless grows to a size similar to that of an adult chimpanzee. Further Antón and Leigh (2003) have found that the cranium of anatomically modern $H$. sapiens can be considered to be paeodomorphic as it retains the same shape of the juvenile $H$. erectus (Mojokerto child).

Most of the skeletal traits that separate us from the apes, namely bipedalism (with the associated modifications of the spine, pelvis, femur and knee), skull and face shape, and dentition, can be explained by the process of neoteny. The "final" mutation, which seems to have occurred in an ancestral species living in Africa, profoundly modified the neural architecture of the human brain, which gave rise to the sudden appearance of our modern symbolic thought and language capacity. Although we do not know precisely how this apparent massive increase in cognitive ability emerged in the human brain, we can determine certain allometric departures in the human brain. Rilling and Seligman (2002) have shown that although the frontal lobes of the human brain are not larger than expected for an ape of our size, the pre-frontal sector is disproportionately large. The human 
temporal lobe white matter volume is significantly expanded to produce a different shape and size with many more connections linking temporal and prefrontal cortex. This extra-allometric expansion appears to be specifically involved with speech production and comprehension necessary for language. Most of this expansion occurs postnatally in humans and a second cycle of development in this region of the brain begins around two years of age in human infants (Pollack, 1994, 165).

\section{The first "Sudden Appearance"}

The earliest fossil hominins dating to around 6-7 million years ago are found in Africa where they existed up until 1.2 million years ago (Lahr \& Foley, 2004). The defining features of these australopithecines were bipedalism, small stature (1-1.5 metres tall) and reduced sized canines. The australopithecines show a mosaic of ape-like and human-like features including tooth size and shape, face shape, brow ridges, brain case size, femur shape, pelvic size and shape and various configurations of foot bones that may mean full bipedalism or some retention of the ability to retain an aboreal, climbing lifestyle.

The most plausible account for the emergence of the first hominin is for a simple heterochronic mutation that changed the developmental pathway of a chimpanzee/s that in particular, did not go through the normal juvenile growth spurt thereby retaining the bipedalism, skull shape and reduced canines of its infancy. This creature, which we can assume had the same, or very close, genetic make-up of its progenerator, may have interbred with its original chimpanzee group to produce most of the variation that we now find in the fossil record over a five million year time span. It is interesting to note that these hominins retained chimpanzeesized brains throughout their approximately 5 million year history (Lahr \& Foley, 2004). Importantly, there are no stone tools associated with australopithecines.

The implications of this stasis for the evolution of human-like cognition and language are quite profound. It is generally agreed that a fundamental requirement for the use of language is the capacity for symbolic thought. Sterelny (2003) stresses the fact that we rely on the symbolic meaning in each of our utterances to be understood by our audience. We need a rich theory of mind for language to operate successfully. Despite many years of experimentation with Great Apes, there is little evidence that they have rich ways of representing the minds of others. We therefore have no reason to suppose that the australopithecines, during their approximately 4-5 million years of existence as a species, had made any major cognitive advance over the Great Apes and were probably not capable of using any sort of proto-language. This conclusion is difficult to reconcile with the notion that language has evolved gradually and incrementally, as Jackendoff and Pinker claim. The more compelling evidence from the paleontological and archaeological record reveals an astonishing stasis for this great expanse of time. 


\section{Homo erectus}

Around 2 million years ago, after around 4-5 million years of stasis, H. erectus, a generally much larger hominin than the australopithecines and the first toolmaker $^{\mathrm{a}}$, first appears in Africa. Shortly after their emergence, they migrated to most other accessible continents. The stone tools found with this species were effectively identical to the first stone tools that appeared in the record 500,000 years earlier (Tattersall, 1997). This suggests that although this early H. erectus population was larger in physical size and cranial capacity than $H$. habilis, it had not developed any notable increase in cognitive ability.

After extensive analysis of the fossil record of most of the H. erectus remains covering Africa, China, Europe and South East Asia, Rightmire (1990) has concluded that this species had maintained a conservative morphology throughout the Pleistocene. H. erectus features vary, but these variations, especially in brain size, were not on a scale greater than those found within contemporary modern human populations. These phenotypic differences can be found within geographically widespread groups of $H$. erectus and cannot be placed within a specific time-line of size or complexity, arguing against a gradual change within groups or arising from intergroup competition. Despite the obvious increase in cranial capacity around 2 million years ago, Walker \& Shipman (1996) argue that any difference in brain size from $H$. habilis to $H$. erectus was simply due to an increase in body size. H. erectus had a body size slightly larger than most modern humans, but nevertheless had only a brain the size of a one year old human.

Pinker (1997) has asserted that an increase in the sophistication of stone tools is one of the keys to understanding the gradual increase in human cognitive complexity. Around 1.4 million years ago, a relatively standardized and symmetrical stone tool was being made in Africa (Tattersall, 1997). We could concede that this probably represents an increase in cognitive ability for our hominin ancestors although even this proposed advance in planning ability has been challenged by some. For example, Ambrose (2001) has suggested that the Acheulian hand-axes are more likely to be the "unintended byproduct of nonstylistic factors rather than intended target types". When raw materials are particularly scarce, a cutting tool would need to be resharpened rather than discarded and we can see how a simple cleaver becomes a teardrop-shaped hand-axe after several bouts of resharpening. Moreover, this tear-drop shaped acheulian hand-axe, known for its distinctive shape, and associated with $H$. erectus, remained unchanged for one million years. It is found wherever $H$. erectus roamed, which covered a distance of 10,000 miles from Africa to Japan. Bickerton asks the question, "is it possible to think of any sapiens innovation that has traveled for the best part of 10 thousand miles without undergoing the slightest change?". He adds that "the mismatch between the

\footnotetext{
aThe first stone tool makers H. habilis, were hardly bigger or more "advanced" in their body skeletons than the australopithecines.
} 
fossil and archaeological records forms an acute embarrassment for those who believe that human cognitive capacities, including language, developed gradually" (Bickerton, 2002, 106).

Pinker and Jackendoff argue that the larynx was recently adapted for speech. MacLarnon \& Hewitt (2004) note that shape of the basicranium of H. erectus indicates that the larynx and hyoid were both situated in the same position as in apes. However Pinker and Jackendoff overlook this fact when claiming that the descended larynx is part of a suite of vocal-tract modifications that were evolutionarily shaped to "subserve" language. There is no gradual evolution of the morphology of the vocal tract that is being "fine-tuned" to serve language.

A further mismatch between advanced anatomy and cognitive behaviour has been found with recent excavations of a 800,000 year old cave site in Atapuerca, Spain. A juvenile hominin fossil has the face of a totally modern human, and Arsuaga (1999) argues that this face may explain how modern humans emerged due to a mutation that caused the retention of juvenile morphology (neoteny) of this ancient species, which he has named Homo antecessor. However, the tools found alongside $H$. antecessor were archaic for their time, resembling the 2.5-2.6 Myr old Oldowan tradition from Africa.

\section{The Appearance of Modern Humans}

The first appearance of anatomically modern humans emerged in Africa by around 120,000 years ago (Stringer, 2003). The fossil record in Africa closely matches the latest genetic evidence for this recent emergence. Pearson (2004) has pointed out that MtDNA and the $\mathrm{X}$ and $\mathrm{Y}$ chromosomes in all modern humans show a very low diversity, especially compared to Apes. It appears that an anatomically modern population emerged in Africa and subsequently replaced all other hominin populations living there at that time. Pearson suggests that these first modern humans slowly grew in population over a period of 60,000 years, but remained in Africa, explaining the greater genetic diversity that is found there today. After 60,000 years, these modern peoples then dispersed to the Near East, South East Asia and Australia, and then on to Europe and the Far East.

Pearson's findings are matched by clear evidence from the archaeological record, that most of what we recognize as fully modern human intentional and symbolic behavior, arose suddenly in southern Africa, around 90,000 to 100,000 years ago (Mellars, 2005). New forms of skin working technology appear along with highly specialized geometric blades, used as insets in multi-component hafted tools, together with intricately shaped barbed bone points. High quality raw materials had been deliberately transported from a distance of at least $20 \mathrm{~km}$. Many sites have an abundance of red ochre showing signs of scraped surfaces indicating their use as coloring pigments. Other large pieces of ochre have been deliberately incised with repetitive geometrical patterns from which we can infer some sort of symbolic or ceremonial activity (Mellars, 2005). Large amounts of 
red ochre are also associated with ceremonial burial. We find an abundance of carefully perforated shells, which again had been imported from long distances. Their use as personal ornaments is confirmed by unambiguous indications of elaborate ceremonial burials associated with a range of perforated seashells (Mellars, 2005). We cannot say whether or not these first humans had language, but we can be sure that they were engaging in symbolic behaviour, making it highly likely that some form of language was at least possible.

\section{Conclusion}

I propose that the archaeological and paleontological record does not support theories that argue for gradual change for the evolution of the hominin clade. Rather, we see the sudden appearance of the first hominins around 6-7 million years ago due to a neotenous mutation of an ancestral ape, followed by an astonishing stasis in bodily form and brain size for at least 4 million years when the first crude stone tools appear. We then find the sudden appearance of $H$. erectus who again shows little variability in either physiology or tool technology for 2.5 million years. It has been shown that any variability within all of these ancestral hominin groups is no more than we find today among modern humans. The final sudden appearance produced anatomically modern humans (H. sapiens), which emerged in Africa around 120,000 years ago with the cognitive architecture to support an extraordinary array of symbolic behaviour not seen before in any ancestral species. I therefore contend that arguments from the gradualist school of thought claiming that our mind and language faculty are highly modularised due to the gradual accretion of functionally specific components, which have evolved gradually over evolutionary time, are not tenable.

\section{References}

Ambrose, S. H. (2001). Paleolithic Technology and Human Evolution. Science, 291, 1746-1753.

Antón, S., \& Leigh, S. (2003). Growth and life history in Homo erectus. In Thompson (2003) (p. 219-245). Cambridge: Cambridge University Press.

Arsuaga, J., Martinez, I., Lorenzo, C., \& Gracia, A. (1999). The Human Cranial Remains from Gran Dolina Lower Pleistocene Site. Journal of Human Evolution, 37, 431-457.

Bickerton, D. (2002). From protolanguage to language. In Crow (2002) (p. 103-120). UK: Oxford University Press.

Crow, T. (2002). The speciation of modern homo sapiens. UK: Oxford University Press.

Jackendoff, R. (1999). Possible Stages in the Evolution of the Language Capacity. Trends in Cognitive Sciences, 3, 272-279.

Knight, C., Studdert-Kennedy, M., \& Hurford, J. (2000). The evolutionary emergence of language. Cambridge, UK: Cambridge University Press. 
Kuykendall, K. (2003). Reconstructing australopithecine growth and development: What do we think we know? In Thompson (2003) (p. 191-218). Cambridge: Cambridge University Press.

Lahr, M. M., \& Foley, R. (2004). Palaeoanthropology: Human Evolution Writ Small. Nature, 431, 1043-1044.

Lovejoy, C., Cohn, M., \& White, T. (1999). Morphological Analysis of the Mammalian Postcranium: A Developmental Perspective. Proc. Natl. Acad. Sci. USA, 96, 13247-13252.

MacLarnon, A., \& Hewitt, G. (2004). Increased Breathing Control: Another Factor in the Evolution of Human Language. Evolutionary Anthropology, 13, 181-197.

Mellars, P. (2005). The Impossible Coincidence. A Single-Species Model for the Origins of Modern Human Behavior in Europe. Evolutionary Anthropology, $14,12-27$.

Pearson, O. (2004). Has the Combination of Genetic and Fossil Evidence Solved the Riddle of Modern Human Origins? Evolutionary Anthropology, 13, 145-159.

Penin, X., Berge, C., \& Baylac, M. (2002). Ontogenetic Study of the Skull in Modern Humans and the Common Chimpanzees: Neotenic Hypothesis Reconsidered with a Tridimensional Procrustes Analysis. American Journal of Physical Anthropology, 118, 50-62.

Pinker, S. (1994). The language instinct. NY: William Morrow \& Co.

Pinker, S. (1997). How the mind works. Australia: Penguin.

Pollack, R. (1994). Signs of life. Boston, US.: Houghton Mifflin.

Rightmire, G. (1990). The evolution of homo erectus. Uk: Cambridge University Press.

Rilling, J., \& Seligman, R. (2002). A quantitative morphometric comparative analysis of the primate temporal lobe. Journal of Human Evolution, 42, 505-533.

Sterelny, K. (2003). Thought in a hostile world. UK: Blackwell.

Stringer, C. (2003). Out of Ethiopia. Nature, 423, 692-694.

Tattersall, I. (1997). Out of Africa Again ... and Again? Scientific American, April, 60-67.

Thompson, J., Krovitz, G., \& Nelson, A. (2003). Patterns of Growth and Development in the Genus Homo. Cambridge: Cambridge University Press.

Walker, A., \& Shipman, P. (1996). The wisdom of the bones. NY: Alfred A. Knopf. 